\title{
New Channel of Plasmon Photovoltaic Effect in Metalized Perovskite Solar Cells
}

\section{Supporting Information}

\author{
Witold A. Jacak and Janusz E. Jacak \\ Department of Quantum Technology, Wroctaw University of Science and Technology, Wroctaw, Poland
}

\section{A. Reducing of exciton binding energy due to relative momentum of e-h pair}

Let us consider electron-hole (e-h) pair with the reduced mass $\mu=\frac{m_{n}^{*} m_{p}^{*}}{m_{n}^{*}+m_{p}^{*}}$, where $m_{n}^{*}$ is the effective mass of electron in the conductivity band and $m_{p}^{*}$ is the hole mass in the valence band. When the relative momentum of the e-h pair, $\hbar \mathbf{k}_{1}-$ $\hbar \mathbf{k}_{2}=\hbar \mathbf{q}$ is nonzero $\left(\hbar \mathbf{k}_{1(2)}\right.$ is a pseudomomentum of electron (hole)), then thus opposite momenta tend to separate the e-h pair. In the simplified model, the stationary state energy of the particle with the mass $\mu$ in the well of mutual Coulomb attraction of electron and hole, $-\frac{e^{2}}{\varepsilon r}$ ( $r$ is the relative distance between electron and hole, $\varepsilon$ is the dielectric permittivity of the semiconductor) cannot be lower than the mean energy of this particle. The mean energy of the particle with mass $\mu$ in this well has the following form,

$$
E(r)=\frac{\hbar^{2}}{2 \mu r^{2}}-\frac{e^{2}}{\varepsilon r},
$$

because at the width $r$ of the well the uncertainty of momentum is $\Delta p \sim \frac{\hbar}{r}$ and the mean kinetical energy cannot be lower than $\frac{h^{2}}{2 \mu r^{2}}$. This averaged energy has a minimum, $E\left(r^{*}\right)=-\frac{e^{4} \mu}{2 \hbar^{2} \varepsilon^{2}}$ at $r^{*}=\frac{\hbar \varepsilon}{e^{2} \mu} . E\left(r^{*}\right)$ is the binding energy of the exciton, whereas $r^{*}$ is the exciton size. If the e-h pair has nonzero relative momentum $\hbar q \neq 0$ (it is the same at any inertial reference system in contrary to the sum of momenta of e and $\mathrm{h}$ ), the minimal energy mean value must be shifted by $\frac{\hbar^{2} q^{2}}{2 \mu}$. The new minimal energy, $E\left(r^{*}\right)+\frac{\hbar^{2} q^{2}}{2 \mu}$, lying higher in the hyperbolic well defines a new larger size of the exciton, $r^{* *}$. For $\frac{\hbar^{2} q^{2}}{2 \mu}=-E\left(r^{*}\right)$ the exciton dissociates $\left(r^{* *} \rightarrow \infty\right)$.

Thus the exciton with nonzero $q$ is less strongly bound and easier dissociates. In the perovskite solar cells, such excitons stronger contribute to the photo-current. In p-n junction-type solar cells the relative momentum of the exciton e is not important because the junction voltages highly exceeds exciton binding energy and all excitons are instantly dissociated regardless their internal momentum structure.

Note that at the ordinary photo-effect only vertical interband transitions are admitted. The reason of it is the form of the perturbation of the electron band system by incident photons. Photons correspond to plane waves, $\mathbf{A}=\mathbf{A}_{0} e^{i(\mathbf{p} \cdot \mathbf{R}-\omega t)}$ (A it a vector potential of an incident e-m wave). The perturbation of the band electron Hamiltonian arises from the kinematical momentum, $\hat{H}=\frac{\left(\hbar \mathbf{k}-\frac{e}{c} \mathbf{A}\right)^{2}}{2 m^{*}} \simeq \frac{\hbar^{2} k^{2}}{2 m^{*}}-\frac{e \hbar \mathbf{k} \cdot \mathbf{A}}{c m^{*}}$ (at gauge, $\nabla \cdot \mathbf{A}=0)$. The perturbation attains thus the form,

$$
\begin{aligned}
& w_{0}(\mathbf{R}, t)=\frac{i e \hbar}{c m^{*}} \cos (\omega t-\mathbf{k} \cdot \mathbf{R}+\alpha)\left(\mathbf{A}_{0} \cdot \nabla\right) \\
& =\frac{i e \hbar}{2 c m^{*}}\left(e^{i(\omega t-\mathbf{k} \cdot \mathbf{R}+\alpha)}+e^{-i(\omega t-\mathbf{k} \cdot \mathbf{R}+\alpha)}\right)\left(\mathbf{A}_{0} \cdot \nabla\right) .
\end{aligned}
$$

Because in this case, all the states of band electrons and of the photon have the form of plane waves, the matrix element in the Fermi golden rule is proportional to the Dirac delta with respect to the momentum sum. This proportionality expresses momentum conservation in a translation invariant system,

$$
\begin{aligned}
& <\mathbf{k}_{1}\left|w_{0}(\mathbf{R}, t)\right| \mathbf{k}_{2}>=\frac{i \hbar e}{2 c m^{*}(2 \pi)^{3}} \int d^{3} R e^{-i\left(\mathbf{k}_{1}+\mathbf{k}\right) \cdot \mathbf{R}}\left(\mathbf{A}_{0} \cdot \nabla\right) e^{i \mathbf{k}_{2} \cdot \mathbf{R}} \\
& =-\frac{e \hbar}{2 c m^{*}} \mathbf{A}_{0} \cdot \mathbf{k}_{2} \boldsymbol{\delta}\left(\mathbf{k}_{1}+\mathbf{k}-\mathbf{k}_{2}\right) .
\end{aligned}
$$

Because of the high value of the photon velocity, $c$, the photon momentum $\hbar \mathbf{k}$ is negligibly small, and thus only vertical transitions, $\hbar \mathbf{k}_{1}=\hbar \mathbf{k}_{2}$, are permitted in the ordinary photo-effect.

However, this is not the case for electron excitations induced by dipole surface plasmons in metallic nanoparticles interacting in the near-field regime with the semiconductor substrate. This system is not translation invariant, and excitations other than vertical ones are permitted, which results in strengthening of inter-band transition probability and generates nonzero relative momentum, $\hbar q \neq 0$, of e-h pairs.

\section{B. Calculation of mean value of relative momentum $\hbar q$ of e-h pairs induced by plasmons}

Perturbation of band electrons by dipole surface plasmon at the metallic nanoparticle is given by Eq. (2) in the main text. This perturbation has an infinite decomposition into the Fourier series which dismisses here the momentum conservation constraint and admits arbitrary pairs of initial, $\mathbf{k}_{1}$, and final, $\mathbf{k}_{2}$, states for transition with nonzero probability.

Let us calculate with all detail the probability (per time unit) of inter-band transitions of electrons in substrate semiconductor induced by dipole plasmons in metallic nano-spheres (with radius $a$ ) embedded in the semiconductor. The surface plasmons couple to the semiconductor band electrons in the nearfield regime. The situation is similar to the ordinary photoeffect, though the perturbation of the electron system in semiconductor is not of a plane-wave form as it was in the case of direct illumination of a semiconductor by incident photons, but attains the form of dipole-type near-field electric interaction, Eq. (2) in the main text, which has infinite Fourier series decomposition and admits transitions between arbitrary $\mathbf{k}_{1}$ and $\mathbf{k}_{2}$ Bloch states. This can be expressed by the matrix element in the relevant Fermi golden rule formula. According 
to Eq. (2) in the main text, the perturbation potential can be written as, [1]:

$$
w^{+}=\left(w^{-}\right)^{*}=\frac{e}{\varepsilon_{0} R^{2}} \frac{e^{i \alpha}}{2 i} \hat{\mathbf{n}} \cdot \mathbf{D}_{0},
$$

corresponding to the emission ( $\hat{\mathbf{n}}=\frac{\mathbf{R}}{R}, \mathbf{D}_{0}$ is the dipole surface plasmon amplitude). The semiconductor band system we model in the simplest single-band parabolic effective mass approximation. The inter-band transition probability is given by the Fermi golden rule,

$$
w\left(\mathbf{k}_{1}, \mathbf{k}_{2}\right)=\frac{2 \pi}{\hbar}\left|<\mathbf{k}_{1}\right| w^{+}\left|\mathbf{k}_{2}>\right|^{2} \delta\left(E\left(\mathbf{k}_{1}\right)-E\left(\mathbf{k}_{2}\right)+\hbar \omega\right),
$$

where Bloch states in the conduction and valence bands we assume as planar waves (for the sake of simplicity),

$$
\begin{aligned}
& \Psi_{\mathbf{k}_{1}}=\frac{1}{(2 \pi)^{3 / 2}} e^{i \mathbf{k}_{1} \cdot \mathbf{R}-i E\left(\mathbf{k}_{1}\right) t / \hbar}, \Psi_{\mathbf{k}_{2}}=\frac{1}{(2 \pi)^{3 / 2}} e^{i \mathbf{k}_{2} \cdot \mathbf{R}-i E\left(\mathbf{k}_{2}\right) t / \hbar}, \\
& E\left(\mathbf{k}_{1}\right)=-\frac{\hbar^{2} \mathbf{k}_{1}^{2}}{2 m_{p}^{*}}-E_{g}, E\left(\mathbf{k}_{2}\right)=\frac{\hbar^{2} \mathbf{k}_{2}^{2}}{2 m_{n}^{*}},
\end{aligned}
$$

(indices $n, p$ refer to electrons from the conduction and valence bands, respectively, $E_{g}$ is the forbidden gap).

The electron wave functions are normalized to the Dirac delta, which corresponds to an infinite movement and the continuous energy spectrum. The wave function modulus squares do not have, in this case, probability interpretation (which must be normalized to the unity), therefore the expression, $w\left(\mathbf{k}_{1}, \mathbf{k}_{2}\right)=\frac{2 \pi}{\hbar}\left|<\mathbf{k}_{1}\right| w^{+}\left|\mathbf{k}_{2}>\right|^{2} \delta\left(E\left(\mathbf{k}_{1}\right)-E\left(\mathbf{k}_{2}\right)+\hbar \omega\right)$, has to be divided by the delta Dirac square, i.e., by the factor, $\left(\frac{V}{(2 \pi)^{3}}\right)^{2}$. This factor corresponds to the probability proper normalization, because,

$$
\frac{1}{(2 \pi)^{3}} \int d^{3} r e^{i \mathbf{k} \cdot \mathbf{r}}=\delta(\mathbf{k}) \simeq(\text { for } \mathbf{k}=0) \frac{V}{(2 \pi)^{3}}(V \rightarrow \infty) .
$$

Note, that the same factor occurs also due to the density of states, when one integrates over all initial and final states, $\mathbf{k}_{1}$ and $\mathbf{k}_{2}$. Two integrals will give the factor $\left(\frac{2 V}{(2 \pi)^{3}}\right)^{2}$, (2 is caused by the spin degeneracy). Thus, both renormalization factors cancel mutually themselves (independently of $V \rightarrow \infty$ ).

Taking into account the above described renormalization, one can find the matrix element,

$$
<\mathbf{k}_{1}\left|w^{+}\right| \mathbf{k}_{2}>=\frac{1}{(2 \pi)^{3}} \int d^{3} R \frac{e}{\varepsilon_{0} 2 i} e^{i \alpha} \hat{\mathbf{n}} \cdot \mathbf{D}_{0} \frac{1}{R^{2}} e^{-i\left(\mathbf{k}_{1}-\mathbf{k}_{2}\right) \cdot \mathbf{R}} .
$$

Let us introduce the vector $\mathbf{q}=\mathbf{k}_{2}-\mathbf{k}_{1}$. One can choose the coordinate system in such a way, that the vector $\mathbf{q}$ is oriented along the $z$ axis, and the vector $\mathbf{D}_{0}$ lies in the plane $z x$ (as is depicted in Fig. S1). Then, $\mathbf{q}=$ $(0,0, q), \hat{\mathbf{n}}=\frac{\mathbf{R}}{R}=\left(\sin \Theta_{1} \cos \psi_{1}, \sin \Theta_{1} \sin \psi_{1}, \cos \Theta_{1}\right), \mathbf{R}=$ $R\left(\sin \Theta_{1} \cos \psi_{1}, \sin \Theta_{1} \sin \psi_{1}, \cos \Theta_{1}\right), \mathbf{D}_{0}=D_{0}(\sin \Theta, 0, \cos \Theta)$ and

$$
\begin{gathered}
\mathbf{q} \cdot \mathbf{R}=q R \cos \Theta_{1}, \\
\hat{\mathbf{n}} \cdot \mathbf{D}_{0}=D_{0}\left(\sin \Theta \sin \Theta_{1} \cos \psi_{1}+\cos \Theta \cos \Theta_{1}\right) .
\end{gathered}
$$

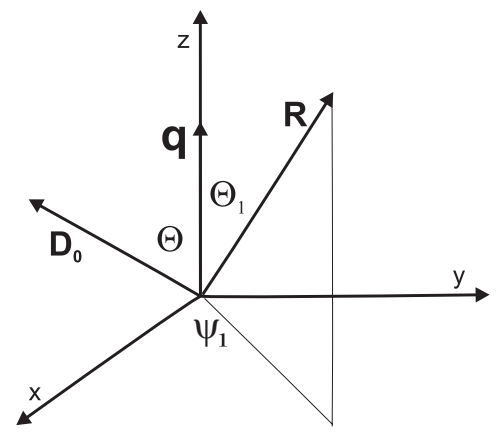

FIG. S1. The reference frame is chosen in the way that the vector $\mathbf{q}$ is oriented along the axis $z$, while the vector $\mathbf{D}_{0}$ lies in the plane $x z$.

Hence,

$$
\begin{aligned}
& <\mathbf{k}_{1}\left|w^{+}\right| \mathbf{k}_{2}> \\
& =\frac{1}{(2 \pi)^{3}} \int d^{3} R \frac{e}{\varepsilon_{0} 2 i} e^{i \alpha} \hat{\mathbf{n}} \cdot \mathbf{D}_{0} \frac{1}{R^{2}} e^{-i\left(\mathbf{k}_{1}-\mathbf{k}_{2}\right) \cdot \mathbf{R}} \\
& =\frac{1}{(2 \pi)^{3}} \frac{e e^{i \alpha}}{\varepsilon_{0} 2 i} D_{0} \int_{b}^{\infty} \frac{R^{2}}{R^{2}} d R \int_{0}^{\pi} \\
& \times \sin \Theta_{1} d \Theta_{1} \int_{0}^{2 \pi} d \psi_{1}\left\{\cos \Theta \cos \Theta_{1}+\sin \Theta \sin \Theta_{1} \cos \psi_{1}\right\} e^{i q R \cos \Theta_{1}} \\
& =\frac{1}{(2 \pi)^{3}} \frac{e e^{i \alpha}}{\varepsilon_{0} 2 i} D_{0} \cos \Theta 2 \pi \int_{a}^{\infty} d R \int_{0}^{\pi} \cos \Theta_{1} \sin \Theta_{1} d \Theta_{1} e^{i q R \cos \Theta_{1}},
\end{aligned}
$$

the integer over $d \psi_{1}$ vanishes the second term in the parenthesis and only the first term contributes with the factor $2 \pi$. Thus,

$$
\begin{aligned}
& <\mathbf{k}_{1}\left|w^{+}\right| \mathbf{k}_{2}>=\frac{-1}{(2 \pi)^{3}} \frac{e e^{i \alpha}}{\varepsilon_{0}} D_{0} \cos \Theta(2 \pi) \int_{b}^{\infty} d R \frac{1}{q} \frac{d}{d R} \frac{\sin q R}{q R} \\
& =\frac{1}{(2 \pi)^{2}} \frac{e e^{i \alpha}}{\varepsilon_{0}} D_{0} \cos \Theta \frac{1}{q} \frac{\sin q b}{q b} \lim _{b \rightarrow 0} \frac{1}{(2 \pi)^{2}} \frac{e e^{i \alpha}}{\varepsilon_{0}} D_{0} \cos \Theta \frac{1}{q} .
\end{aligned}
$$

The lower limit of the integral with respect to $R$ is taken as $b$ (the external radius of the dielectric coating, the onset of the semiconductor). In this manner one can constrain the accessible space for planar waves in the semiconductor for a completely embedded nano-sphere in the surrounding material. This is, however, a crude approximation as tunneling effect certainly would allow an access to the inner nano-particlespace, to some extent, at least.

Now we will integrate over all initial and final states of both bands. The related integration over $\mathbf{k}_{1}, \mathbf{k}_{2}$, one can substitute with integration over $\mathbf{q}, \mathbf{k}_{2}$ (Jacobian is -1 ). Scalar products are invariant against coordinate systems rotations, therefore the result of integration will be the same if $\mathbf{q}$ was along $z$ axis or $\mathbf{D}_{0}$ is oriented now along the $z$ axis- $\Theta$ gives, in the latter case, the deviation of $\mathbf{q}$ with respect to $z$ direction-this choice of the reference frame is convenient for integration with respect to $d^{3} q$.

Thus we arrive with the formula for transition probability in the following form,

$$
\delta w=\int d^{3} k_{1} \int d^{3} k_{2}\left[f_{1}\left(1-f_{2}\right) w\left(\mathbf{k}_{1}, \mathbf{k}_{2}\right)-f_{2}\left(1-f_{1}\right) w\left(\mathbf{k}_{2}, \mathbf{k}_{1}\right)\right],
$$

where, $f_{1}, f_{2}$ assign the temperature dependent distribution functions (Fermi-Dirac distribution functions) for initial and final states, respectively. The emission and absorption were 
included in the overall transition probability, but for room temperatures one can assume, $f_{2} \simeq 0$ and $f_{1} \simeq 1$, which leads to,

$$
\delta w=\int d^{3} k_{1} \int d^{3} k_{2} w\left(\mathbf{k}_{1}, \mathbf{k}_{2}\right) .
$$

In the above formula we avoided the density state factors canceled by the probability renormalization, as was mentioned above.

We must thus calculate the following integrals,

$$
\begin{aligned}
& \delta w=\int d^{3} k_{2} \int d^{3} q \frac{e^{2}}{(2 \pi)^{3} \hbar \varepsilon} \frac{D_{0}^{2} \cos ^{2} \Theta}{q^{2}} \frac{\sin ^{2}(q b)}{(q b)^{2}} \\
& \times \delta\left(\frac{\hbar^{2} k_{1}^{2}}{2 m_{n}^{*}}+\frac{\hbar^{2} k_{2}}{2 m_{p}^{*}}-\left(\hbar \omega-E_{g}\right)\right), \\
& \delta w_{1}=\int d^{3} k_{2} \int d^{3} q \frac{e^{2}}{(2 \pi)^{3} \hbar \varepsilon} \frac{\hbar^{2} q^{2}}{2 \mu} \frac{D_{0}^{2} \cos ^{2} \Theta}{q^{2}} \frac{\sin ^{2}(q b)}{(q b)^{2}} \\
& \times \delta\left(\frac{\hbar^{2} k_{1}^{2}}{2 m_{n}^{*}}+\frac{\hbar^{2} k_{2}}{2 m_{p}^{*}}-\left(\hbar \omega-E_{g}\right)\right),
\end{aligned}
$$

with

$$
\begin{aligned}
& \delta\left(\frac{\hbar^{2} k_{1}^{2}}{2 m_{n}^{*}}+\frac{\hbar^{2} k_{2}}{2 m_{p}^{*}}-\left(\hbar \omega-E_{g}\right)\right) \\
& =\frac{1}{\alpha+\beta} \frac{1}{2 \beta^{\prime} q k_{2}} \delta\left(\cos \Theta_{2}-\frac{k_{2}^{2}+\beta^{\prime} q^{2}-\gamma^{\prime}}{2 \beta^{\prime} q k_{2}}\right),
\end{aligned}
$$

where $\alpha=\frac{\hbar^{2}}{2 m_{n}^{*}}, \beta=\frac{\hbar^{2}}{2 m_{p}^{*}}, \gamma=\hbar \omega-E_{g}, \alpha^{\prime}=\frac{\alpha}{\alpha+\beta}, \beta^{\prime}=\frac{\beta}{\alpha+\beta}$, and $\gamma^{\prime}=\frac{\gamma}{\alpha+\beta}, \mu=\frac{m_{n}^{*} m_{p}^{*}}{m_{n}^{*}+m_{p}^{*}}$. For each integral with respect to $\mathbf{k}_{2}$ and $\mathbf{q}$, the coordinate system can be rotated independently, and for integration over $d \mathbf{k}_{2}$, we again choose the orientation of $\mathbf{q}$ along the $z$ axis, which leads to the spherical angle $\Theta_{2}$ resulting from the product, $\mathbf{k}_{2} \cdot \mathbf{q}=k_{2} q \cos \Theta_{2}$. The expressions for $\delta w$ and $\delta w_{1}$ thus attain the form,

$$
\begin{aligned}
& \delta w=\frac{e^{2} D_{0}^{2}}{(2 \pi)^{3} \hbar \varepsilon^{2}} \int d^{3} q^{\sin ^{2}(q b)} \frac{\cos \Theta}{q^{2} b^{2}} \frac{q^{2}}{q^{2}} \\
& \int_{0}^{\infty} d k_{2} k_{2}^{2} \int_{0}^{\pi} d \Theta_{2} \sin \Theta_{2} \\
& \times \int_{0}^{2 \pi} d \psi_{2} \frac{1}{\alpha+\beta} \frac{1}{2 \beta^{\prime} q k_{2}} \delta\left(\cos \Theta_{2}-\frac{k_{2}^{2}+\beta^{\prime} q^{2}-\gamma^{\prime}}{2 \beta^{\prime} q k_{2}}\right), \\
& \delta w_{1}=\frac{e^{2} D_{0}^{2}}{(2 \pi)^{3} \hbar \varepsilon^{2}} \int d^{3} q \frac{\sin ^{2}(q b)}{q^{2} b^{2}} \frac{\hbar^{2} \cos \Theta}{2 \mu} \\
& \int_{0}^{\infty} d k_{2} k_{2}^{2} \int_{0}^{\pi} d \Theta_{2} \sin \Theta_{2} \\
& \times \int_{0}^{2 \pi} d \psi_{2} \frac{1}{\alpha+\beta} \frac{1}{2 \beta^{\prime} q k_{2}} \delta\left(\cos \Theta_{2}-\frac{k_{2}^{2}+\beta^{\prime} q^{2}-\gamma^{\prime}}{2 \beta^{\prime} q k_{2}}\right) .
\end{aligned}
$$

The integration over $d \Theta_{2}$ involves the Dirac delta. The relevant nonzero contribution (arising from integration over $\left.d \cos \Theta_{2}\right)$ is conditioned by the inequalities (as $\left|\cos \left(\Theta_{2}\right)\right| \leq 1$ ),

$$
-1 \leq \frac{k_{2}^{2}+\beta^{\prime} q^{2}-\gamma^{\prime}}{2 \beta^{\prime} q k_{2}} \leq 1
$$

which manifest as the following constraints:

$$
q \leq \xi,
$$

where $\xi=\sqrt{\frac{\gamma^{\prime}}{\beta^{\prime}\left(1-\beta^{\prime}\right)}}=\frac{\sqrt{2\left(\hbar \omega-E_{g}\right)\left(m_{n}^{*}+m_{p}^{*}\right)}}{\hbar}$, and

$$
k_{-} \leq k_{2} \leq k_{+},
$$

with $k_{ \pm}=\left|\beta^{\prime} q \pm \sqrt{\gamma^{\prime}-\left(1-\beta^{\prime}\right) \beta^{\prime} q^{2}}\right|$. Thus,

$$
\begin{gathered}
\delta w=\frac{1}{(\alpha+\beta) 2 \beta^{\prime}} \frac{e^{2} D_{0}^{2}}{2 \pi \hbar \varepsilon^{2}} \int_{0}^{\xi} d q q^{2} \frac{\sin ^{2} q b}{(q b)^{2}} \\
\times \int_{0}^{\pi} d \Theta \sin \Theta \cos ^{2} \Theta \frac{1}{q^{3}} \int_{k_{-}}^{k_{+}} d k_{2} k_{2}, \\
\delta w_{1}=\frac{1}{(\alpha+\beta) 2 \beta^{\prime}} \frac{\hbar^{2} e^{2} D_{0}^{2}}{4 \pi \hbar \varepsilon^{2} \mu} \int_{0}^{\xi} d q q^{4} \frac{\sin ^{2} q b}{(q b)^{2}} \\
\times \int_{0}^{\pi} d \Theta \sin \Theta \cos ^{2} \Theta \frac{1}{q^{3}} \int_{k_{-}}^{k_{+}} d k_{2} k_{2},
\end{gathered}
$$

and hence,

$$
\begin{gathered}
\delta w=\frac{2}{3} \frac{\sqrt{\gamma^{\prime}}}{\alpha+\beta} \frac{e^{2} D_{0}^{2}}{2 \pi \hbar \varepsilon^{2}} \xi \int_{0}^{1} d x \frac{\sin ^{2}(x(b \xi))}{x^{2}(b \xi)^{2}} \sqrt{1-x^{2}} . \\
\delta w_{1}=\frac{2}{3} \frac{\sqrt{\gamma^{\prime}}}{\alpha+\beta} \frac{\hbar^{2} e^{2} D_{0}^{2}}{2 \pi \hbar \varepsilon^{2} 2 \mu} \xi \int_{0}^{1} d x \frac{\sin ^{2}(x(b \xi))}{b^{2}} \sqrt{1-x^{2}} .
\end{gathered}
$$

Therefore,

$$
\begin{aligned}
& \delta w=\frac{4}{3} \frac{\mu^{2}\left(m_{n}^{*}+m_{p}^{*}\right) 2\left(\hbar \omega-E_{g}\right) e^{2} D_{0}^{2}}{\sqrt{m_{n}^{*} m_{p}^{*} 2 \pi \hbar^{5} \varepsilon^{2}}} \int_{0}^{1} d x \frac{\sin ^{2}(x b \xi)}{(x b \xi)^{2}} \sqrt{1-x^{2}} \\
& =\frac{4}{3} \frac{\mu^{2}}{\sqrt{m_{n}^{*} m_{p}^{*}}} \frac{e^{2} D_{0}^{2}}{2 \pi \hbar^{3} \varepsilon^{2}} \xi^{2} \int_{0}^{1} d x \frac{\sin ^{2}(x b \xi)}{(x b \xi)^{2}} \sqrt{1-x^{2}} . \\
& \delta w_{1}=\frac{4}{3} \frac{\hbar^{2} \mu^{2}\left(m_{n}^{*}+m_{p}^{*}\right) 2\left(\hbar \omega-E_{g}\right) e^{2} D_{0}^{2}}{\sqrt{m_{n}^{*} m_{p}^{*} 2 \pi \hbar^{5} \varepsilon^{2} 2 \mu}} \int_{0}^{1} d x \frac{\sin ^{2}(x b \xi)}{b^{2}} \sqrt{1-x^{2}} \\
& =\frac{4}{3} \frac{\hbar^{2} \mu^{2}}{\sqrt{m_{n}^{*} m_{p}^{*}}} \frac{e^{2} D_{0}^{2}}{2 \pi \hbar^{3} \varepsilon^{2} 2 \mu} \xi^{2} \int_{0}^{1} d x \frac{\sin ^{2}(x b \xi)}{b^{2}} \sqrt{1-x^{2}} .
\end{aligned}
$$

Hence, finally,

$$
\Delta E(b)=<\frac{\hbar^{2} q^{2}}{2 \mu}>=\frac{\delta w_{1}}{\delta w}=\frac{\frac{\hbar^{2}}{2 \mu} \int_{0}^{1} d x \frac{\sin ^{2}(x b \xi)}{b^{2}} \sqrt{1-x^{2}}}{\int_{0}^{1} d x \frac{\sin ^{2}(x b \xi)}{(x b \xi)^{2}} \sqrt{1-x^{2}}} .
$$

This final relatively simple formula defines the exciton binding energy reduction due to interaction with plasmons, $\Delta E$ which is dependent of the radius $a$ of the nanosphere via Eq. (S28). This dependence is plotted in Fig. 2 in the main text for $\Delta E(b) /\left(\frac{h^{2} q_{0}^{2}}{2 \mu}\right)$ (normalized by the band energy at the border of the Brillouin zone, i.e., at $q_{0} \sim \frac{\pi}{l}$, where $l \simeq 5 \mathrm{~nm}$ is an averaged size of the elementary cell in perovskite).

\section{Absorption strengthening by plasmon mediation}

The energy out-flow from plasmons to the nearby semiconductor causes their strong damping. The damping of plasmons is thus a measure of the efficiency of plasmon-mediated photo-effect with regard to sun-light energy absorption. The energy in-flow to the nearby semiconductor band system, $\mathscr{A}$, is equal to the out-flow of energy of plasmons oscillating in metallic nanoparticles. Such an assumption is needed for assessment of plasmon damping rate in an idealized situation when the source of energy is only plasmon formerly excited (i.e., without incident sun light). Considering the gradual 
damping of plasmon according to the exponential dipole amplitude lowering, $D_{0}(t)=D_{0} e^{-t / \tau^{\prime}}$, we can estimate the transferred energy by the following formula,

$$
\begin{aligned}
& \mathscr{A}=\beta \int_{0}^{\infty} \delta w \hbar \omega d t=\beta \hbar \omega \delta w \tau^{\prime} / 2 \\
& =\left\{\begin{array}{l}
\frac{2}{3} \frac{\beta \omega \tau^{\prime} \mu \sqrt{m_{n}^{*} m_{p}^{*}}\left(\hbar \omega-E_{g}\right) e^{2} D_{0}^{2}}{\hbar^{4} \varepsilon^{2}}, \text { for } b \xi \ll 1, \\
\frac{2}{3} \frac{\beta \omega \tau^{\prime} \mu^{3 / 2} \sqrt{2} \sqrt{\hbar \omega-E_{g}} e^{2} D_{0}^{2}}{a \hbar^{3} \varepsilon^{2}}, \text { for } b \xi \gg 1,
\end{array}\right.
\end{aligned}
$$

note, that according to the above derivation $\varepsilon$ is the permittivity of the perovskite, which is ca. 5 (by $\varepsilon_{1}$ we denote the permittivity of the dielectric coating of the metallic nanosphere, for $\mathrm{SiO}_{2}$ it is ca. 3.9).

In the above formula the symbol $\tau^{\prime}$ indicates the damping time-rate of surface plasmons coupled to the electrons in the semiconductor substrate. Special attention should be paid to two limiting situations, $\xi b \ll 1$ (small metallic nanoparticles, with radius not larger than $2-3 \mathrm{~nm}$ ), and $\xi b \gg 1$ (large nanoparticles, of radius larger than ca. $5 \mathrm{~nm}$ ). To be specific, let us assess the parameter $\xi$ for considered by us perovskite solar cell. Taking into account the material parameters for perovskite as listed in the Table S3, we find,

$$
\xi=\frac{\sqrt{2\left(\hbar \omega-2.56 \times 10^{-19}\right)\left(18.2 \times 10^{-31}\right)}}{\hbar}[1 / \mathrm{m}],
$$

where $\hbar=1.05 \times 10^{-34} \mathrm{Js}, \omega$ is plasmon circular frequency in units $1 / \mathrm{s}$.

Via the direct comparison of $\mathscr{A}$ given by Eq. (S29) with the initial energy of the plasmon which has been next transferred step-by-step to the semiconductor, one can find the damping rate, $\frac{1}{\tau^{\prime}}$, for plasmons in the considered case. The initial energy of plasmons equals to, $\mathscr{B}=\frac{D_{0}^{2}}{2 \varepsilon a^{3}}$ (important is here the radius $a$ of the metallic core). Assuming that $\mathscr{A}=\mathscr{B}$ we arrive with the formula for the damping rate for plasmons,

$$
\frac{1}{\tau^{\prime}}=\left\{\begin{array}{l}
\frac{4 \beta \omega \mu \sqrt{m_{n}^{*} m_{p}^{*}}\left(\hbar \omega-E_{g}\right) e^{2} a^{3}}{3 \hbar^{4} \varepsilon}, \text { for } b \xi \ll 1, \\
\frac{4 \beta \omega \mu^{3 / 2} \sqrt{2} \sqrt{\hbar \omega-E_{g}} e^{2} a^{2}}{3 \hbar^{3} \varepsilon}, \text { for } b \xi \gg 1 .
\end{array}\right.
$$

By $\tau^{\prime}$ we denote here a large damping of plasmons due to the energy transfer to the semiconductor substrate highly exceeding the internal damping, characterized by $\tau$, the latter due to the scattering of electrons inside the metallic nanoparticle $\left(\frac{1}{\tau} \ll \frac{1}{\tau^{\prime}}\right)$. We neglect also the irradiation to far-field zone of the plasmon energy due to the Lorentz friction, which is also smaller than near-field zone energy transfer to the nearby semiconductor.

If we consider, nanospheres of $\mathrm{Au} / \mathrm{SiO}_{2}$ embedded inside the perovskite layer we obtain for $\omega=\omega_{1}$ (Mie self-frequency of plasmon),

$$
\frac{1}{\tau^{\prime} \omega_{1}}=\left\{\begin{array}{l}
0.54 \beta\left(\frac{a[n m]}{1[n m]}\right)^{3} \frac{\mu}{m} \frac{\sqrt{m_{n}^{*} m_{p}^{*}}}{m}, \text { for } b \xi \ll 1, \\
0.165 \beta\left(\frac{a[n m]}{1[n m]}\right)^{2}\left(\frac{\mu}{m}\right)^{3 / 2}, \text { for } b \xi \gg 1,
\end{array}\right.
$$

for carriers in perovskite, $m_{n}^{*}=1 \mathrm{~m}, m_{p}^{*}=1 \mathrm{~m}, \mathrm{~m}$ is the bare electron mass, $\mu=\frac{m_{n}^{*} m_{p}^{*}}{m_{n}^{*}+m_{p}^{*}}=m / 2$ and $E_{g}=1.6 \mathrm{eV}, \hbar \omega_{1}=2.72$ $\mathrm{eV}, \varepsilon \sim 5$. For these parameters and nanospheres with the radius $a$ in the range of $10-100 \mathrm{~nm}$, the lower case of Eq. (S31) applies (at $\omega=\omega_{1}$ ).

In another scenario (as in the case of a solar cell metallically modified) when the out-flow of the plasmon energy is recovered by the continuous income from the sun-light, one can consider the energy-balanced regime. When whole the incoming to plasmon energy of the monochromatic $\omega$ e-m wave of incident photons is transferred to the semiconductor via the plasmon-electrons coupling channel, we deal with the stationary state of a driven and damped oscillator for plasmons. Even though the free undamped plasmon has the Mie self-resonance frequency, $\omega_{1}=\frac{\omega_{p}}{\sqrt{3 \varepsilon_{1}}}$ (because for sufficiently thick dielectric coating the Mie frequency is accommodated to the dielectric coating material, rather than of perovskite), the frequency of plasma oscillations equals to the driven electric field frequency, $\omega$, of the incident e-m wave of photons. Because of an instant leakage of the plasmon energy in near field-zone to the semiconductor substrate, this large damping of plasmon causes a red-shift and a widening of the resonance, as for conventional damped and driven oscillator. The widened resonance enables the energy transfer from plasmons to electrons to embrace also frequencies lower or larger than Mie frequency but limited from below by the semiconductor forbidden gap $E_{g} / \hbar$.

The total efficiency of the plasmon mediated photo-effect corresponds to the integration over all Fourier components, $\omega>E_{g} / \hbar$, of light interfered with intensity distribution in sun-light spectrum. To optimize the photovoltaic gain the coincidence of sun-light spectrum intensity with spectral characteristic of plasmon transfer efficiency is required. To model this spectral coincidence it is necessary to consider separately each single monochromatic e-m mode, i.e., a Fourier component $\omega$. At this frequency, plasmons are damping with the rate $\frac{1}{\tau^{\prime}}(\mathrm{S} 30)$. This damping causes a red-shift of the resonance and reduces the resonance amplitude, which in turn allows for the accommodation to the balance of energy transfer to the semiconductor with the incident sun-light e-m wave energy intensity (defined by its electric field amplitude $E_{0}$ ) at the frequency $\omega$. Within this damped and driven oscillator model, the accommodated amplitude of plasmon oscillations $D_{0}(\omega)$ is constant in time and shaped by the formula, $f(\omega)=\frac{1}{\sqrt{\left(\omega_{1}^{2}-\omega^{2}\right)^{2}+4 \omega^{2} / \tau^{\prime 2}}}$. The extreme of red-shifted resonance is attained at $\omega_{m}=\omega_{1} \sqrt{1-2\left(\omega_{1} \tau^{\prime}\right)^{-2}}$ with the corresponding amplitude $\sim \tau^{\prime} /\left(2 \sqrt{\omega_{1}^{2}-\tau^{\prime}-2}\right)$. The red-shift is proportional to $1 /\left(\omega_{1} \tau^{\prime 2}\right)$. In the case of the described energy transfer balance one obtains according to Eqs (8) and (10) in 
the main text,

$q_{m}=\left\{\begin{array}{l}\beta C_{0} \frac{128}{9} \pi^{2} b^{3} \frac{\mu \sqrt{\mu_{n}^{*} \mu_{p}^{*}}}{m^{2}}\left(\hbar \omega-E_{g}\right) \frac{e^{6} n_{e}^{2} \omega}{\hbar^{4} \varepsilon^{3}} f^{2}(\omega), \text { for } b \xi \ll 1, \\ \beta C_{0} \frac{128}{9} \sqrt{2} \pi^{2} b^{2} \frac{\mu^{3 / 2}}{m^{2}} \sqrt{\hbar \omega-E_{g}} \frac{e^{6} n_{e}^{2} \omega}{\hbar^{3} \varepsilon^{3}} f^{2}(\omega), \text { for } b \xi \gg 1,\end{array}\right.$

where, $f(\omega)=\frac{1}{\sqrt{\left(\omega_{1}^{2}-\omega^{2}\right)^{2}+4 \omega^{2} / \tau^{\prime 2}}}$ corresponds to the amplitude factor for the driven damped oscillator and $D_{0}=$ $\frac{e^{2} n_{e} E_{0} 4 \pi b^{3}}{3 m} f(\omega)$ (in Eq. (8) in the main text; the amplitude of the electric field, $E_{0}$, in the incident e-m wave is next ruled out from Eq. (S32) due to normalization per single photon as in Eq. (10) in the main text; $C_{0}=\frac{N 4 / 3 \pi a^{3}}{V}, V$ is the volume of the semiconductor $(V=S H, S$ is the cell surface, $H$ is the thickness of perovskite layer), $N$ is the number of metallic nanospheres.

The ratio, $\frac{q_{m}}{q_{0}}$, revealing the advantage of the plasmon mediated photo-effect over the ordinary photo-effect can be expressed as follows,

$$
\frac{q_{m}}{q_{0}}=\left\{\begin{array}{l}
\frac{4 \sqrt{2} \pi^{2} b^{3} \beta C_{0} \sqrt{m_{n}^{*} m_{p}^{*}}\left(m_{p}^{*}\right)^{2} e^{4} n_{e}^{2} \omega^{2} f^{2}(\omega)}{3 \mu^{3 / 2} m^{2} \sqrt{\hbar \omega-E_{g}} \hbar \varepsilon^{2}}, \text { for } b \xi \ll 1, \\
\frac{8 \pi^{2} b^{2} \beta C_{0}\left(m_{p}^{*}\right)^{2} e^{4} n_{e}^{2} \omega^{2} f^{2}(\omega)}{3 \mu m^{2}\left(\hbar \omega-E_{g}\right) \varepsilon^{2}}, \text { for } b \xi \gg 1 .
\end{array}\right.
$$

One can estimate the photo-current in the case of a semiconductor photodiode with metallic plasmonic components without including the discussed in previous paragraphs reduction of the binding energy of excitons, i.e., we can calculate here only net photocurrent enhancement due to only absorption strengthening by plasmons. This photo-current is given by, $I^{\prime}=|e| N_{p}\left(q_{0}+q_{m}\right) A$, where $N_{p}$ is the number of incident photons and $q_{0}$ and $q_{m}$ are the probabilities of single photon absorption in the ordinary photo-effect [2] and of single photon absorption mediated by the presence of metallic nanospheres, respectively, as derived in the previous paragraph. $A=\frac{\tau_{f}^{n}}{t_{n}}+\frac{\tau_{f}^{p}}{t_{p}}$ is the amplification factor $\left(\tau_{f}^{n(p)}\right.$ is the annihilation time of both sign carriers, $t_{n(p)}$ is the drive time for carriers [the time of traversing the distance between the electrodes]). Note, that we included simultaneously absorption of photons directly in the semiconductor later, $q_{0}$, and with mediation of plasmons, $q_{m}$. From the above formulae, it follows that (here $I=I^{\prime}\left(q_{m}=0\right)$, i.e., the photo-current without metallic modifications),

$$
\frac{I^{\prime}}{I}=1+\frac{q_{m}}{q_{0}}
$$

where the ratio $q_{m} / q_{0}$ is given by Eq. (S33).

Note that collective reflection-interference type corrections worsening light absorption are rather not strong for the considered low densities of metallic coverings and nano-sphere sizes well lower than the resonant wave-length, though for larger concentrations and larger nano-sphere sizes, would play a stronger reducing role (reflecting incident sun-light photons) $[3,4]$.

\begin{tabular}{l|l|l}
\hline metal & bulk pl $[\mathrm{eV}]$ & $\begin{array}{l}\text { surface pl in NPs } \\
{[\mathrm{eV}]}\end{array}$ \\
\hline $\mathrm{Li}$ & 6.6 & 3.4 \\
$\mathrm{Na}$ & 5.4 & 3.3 \\
$\mathrm{~K}$ & 3.8 & 2.4 \\
$\mathrm{Mg}$ & 10.7 & 6.7 \\
$\mathrm{Al}$ & 15.1 & 8.8 \\
$\mathrm{Fe}$ & 10.3 & 5.0 \\
$\mathrm{Cu}$ & 6 & 3.5 \\
$\mathrm{Ag}$ & 3.8 & 3.5 \\
$\mathrm{Au}$ & 4.67 & 2.7 \\
\hline
\end{tabular}

TABLE S1. Plasmon energies measured in metals

\begin{tabular}{l|l|l|l}
\hline metal & $\mathrm{Au}$ & $\mathrm{Ag}$ & $\mathrm{Cu}$ \\
\hline Mie frequency $[1 / \mathrm{s}]$ & $4.11 \times 10^{15}$ & $5.2 \times 10^{15}$ & $5.7 \times 10^{15}$ \\
\hline
\end{tabular}

TABLE S2. Mie frequency $\omega_{1}$ to formula (S33)

\section{Experimental comparison of plasmon photo-voltaic effect in perovskite cell doped with core-shell metallic nanoparticles and Si metalized cell}

In the main text we demonstrated that plasmons cause modification both of photon absorption and of local electricity inside the cell metallically doped in nano-scale. Both these channels influence the overall efficiency of the cell with the ultimate value being the subject of the trade-off between competing factors in dependence of material parameters and metallic nanoparticle size and deposition type. In the case of hybrid chemical perovskite cell the second channel occurs to be dominating which is confirmed experimentally [5]. This channel does not concern photon absorption strengthening by plasmons (which is poor in perovskite), but corresponds to strong decrease of binding energy of excitons generated by the plasmon potential in its near field-zone. The lowering of exciton binding energy results in the easier dissociation of excitons at the surface of porous basis of the perovskite layer and in the increase of a photo-current. The experimentally observed significant increase of the photo-current (but not of the voltage) and the eventual efficiency of the perovskite cell growth induced by metallic components is observed, cf. Fig. $\mathrm{S} 2$. The difference with the plasmon effect with the dominating channel of absorption growth (as in the case of Si cell covered by metallic nanoparticles on the cell top, or in other $p-n$ junction solar cells) reflexes different mechanisms of plasmon photo-voltaic effect in perovskite and $p-n$ junction cells. The absorption channel causes an increase both of current and voltage as in Si case-cf. Fig. S3(b,c), whereas the reducing of exciton binding energy strengthens the photocurrent only—cf. Fig. S2(c).

[1] L. D. Landau and E. M. Lifshitz, Field Theory, Nauka, Moscow, 1973.

[2] P. S. Kiriejew, Physics of Semiconductors, PWN, Warsaw, 1969. 

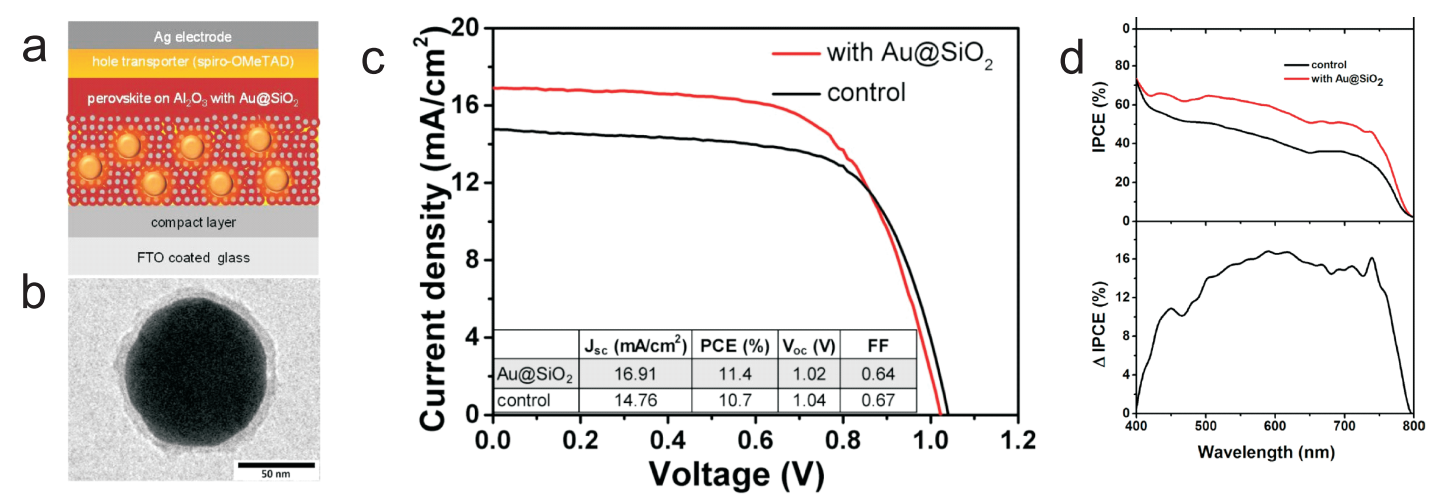

FIG. S2. (a) Illustration of device structure with the component-layers indicated (b) TEM image of Au/SiO 2 core-shell NP $(a \sim 40 \mathrm{~nm}, b \sim 48$ $\mathrm{nm}$-cf. Fig. 1 in the main text. (c) I-V curve for perovskite cell with porous $\mathrm{Al}_{2} \mathrm{O}_{3}$ basis filled with $\mathrm{Au} / \mathrm{SiO}{ }_{2}$ core-shell nanoparticles with density $\sim 1 \mathrm{wt} \%$; a strong increase of the photo-current is noticeably with simultaneous lowering of the voltage. (d) IPCE (photon-to-current conversion efficiency) spectra of control and with $\mathrm{Au} / \mathrm{SiO} 2$ devices and its increase ( $\triangle \mathrm{IPCE}$ ) due to the addition of $\mathrm{Au} / \mathrm{SiO} \mathrm{N}_{2} \mathrm{NPs}(\mathrm{devices}$ measured under AM1.5 simulated sunlight (100 mW/ $\mathrm{cm}^{2}$ irradiance). After experiment [5].
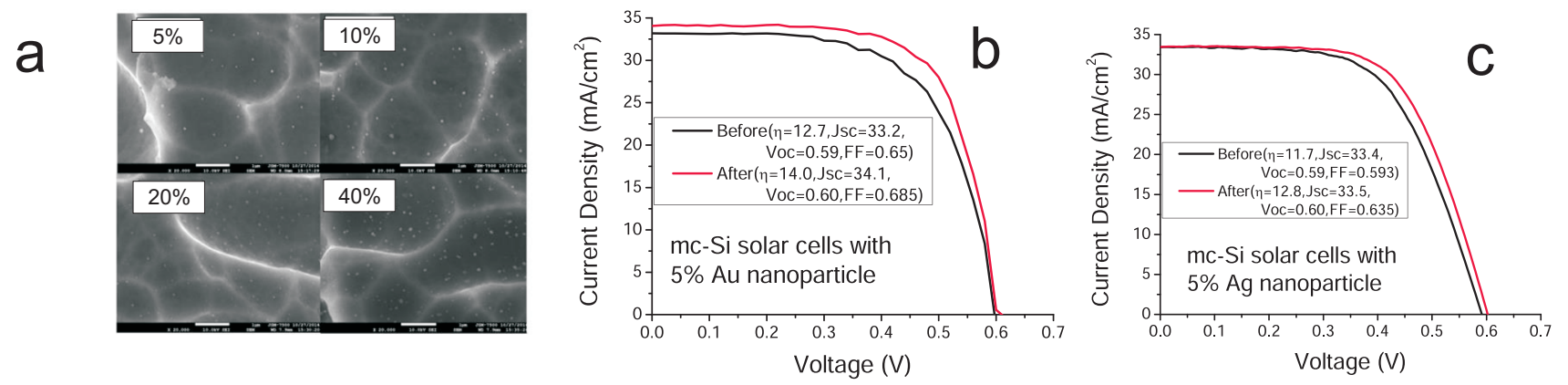

FIG. S3. (a) Visualization by EM photography of distribution of metallic nanoparticles (Au) on the surface of the multi-crystalline Si solar cell under study for plasmonic effect as shown in (b); the very low surface concentration of metallic components is visible (percentage refers to concentration of colloidal solution used for spin coating of a cell surface). (b and c) Comparison of solar cell efficiency due to plasmon modification for the multi-crystal Si solar cell expressed by an increase of the area in the figure beneath the red I-V curve with respect to the area ranged by the black curve, (b) with photoactive surface modified by Au nanoparticles (efficiency increase is ca. 6.5\%), (c) by Ag nanoparticles (efficiency increase is ca. 2.5\%), acc. to our study [4]. The significant difference in I-V characteristic for metalized Si and perovskite cells is noticeable — compare Fig. S2(c)—which corresponds to different mechanism of plasmon induced efficiency increase, by strengthening of the photo-current due to easier dissociation of excitons in perovskite cell.

\begin{tabular}{l|l|l|l}
\hline semiconductor & $m_{n}^{*}$ & $m_{p}^{*}$ & $E_{g}$ \\
\hline perovskite & $1 m$ & $1 m$ & $1.6 \mathrm{eV}$ \\
$\mathrm{CH}_{3} \mathrm{NH}_{3} \mathrm{PbI}_{3}$ & & & \\
$\mathrm{Si}$ & $0.19 m \mathrm{~T}[110]$ & $0.16 m \mathrm{lh}$ & $1.12 \mathrm{eV}$ \\
\hline
\end{tabular}

TABLE S3. Substrate material parameters to formula (S33) $(m=$ $9.1 \times 10^{-31} \mathrm{~kg}$, the mass of bare electron; lh-light holes, Ttransverse)

[3] M. Losurdo, M. M. Giangregorio, G. V. Bianco, A. Sacchetti, P. Capezzuto, and G. Bruno, "Enhanced absorption in $\mathrm{Au}$
nanoparticles/a-Si:H/c-Si heterojunction solar cells exploiting au surface plasmon resonance," Sol. Energy Mater. Sol. Cells 93, p. $1749,2009$.

[4] M. Jeng, Z. Chen, Y. Xiao, L. Chang, J. Ao, Y. Sun, E. Popko, W. Jacak, and L. Chow, "Improving Efficiency of Multicrystalline Silicon and CIGS Solar Cells by Incorporating Metal Nanoparticles," Materials 8(10), pp. 6761 - 6771, 2015.

[5] W. Zhang, M. Saliba, S. Stranks, Y. Sun, X. Shi, U. Wiesner, and H. Snaith, "Enhancement of perovskite-based solar cells employing core-shell metal nanoparticles," Nano Lett. 13(9), p. 4505, 2013. 